\title{
Configuration design and performance analysis of a force feedback operating rod based on 3-RRR spherical parallel mechanism
}

\author{
Zhiming Wei ${ }^{a}$, Dongtao Ma ${ }^{b}$, Anping Tuo ${ }^{c}$, Faduo $\mathrm{Xu}^{\mathrm{d}}$ \\ Lanzhou Institute of Physics, Lanzhou 730000, China \\ acastwzm@163.com, b463814290@qq.com, cTuoap@163.com, d1312186559@qq.com
}

Keywords: 3-RRR spherical mechanism, Force feedback Operating rod, dexterity Task space.

\begin{abstract}
According to the design requirements that the operating rod should provide a sensing capability to make the operator at the remote end be personally on the scene, in this paper, we propose a force feedback operating rod equipped with 3-RRR spherical parallel mechanism as the basic configuration by analyzing the configuration of a variety of operating rods. Through the analysis of the forward and inverse kinematics and statics of the 3-RRR spherical parallel mechanism, we determined the force / torque mapping calculation method of the static calculation, joint space and operation space of the force feedback operating rod. On this basis, taking the mechanism dexterity determined by Gosselin method as an indicator, we integrated the size of force feedback operating rod of 3-RRR mechanism. Finally, through the simulation analysis of the force transmission performance, we proved that the mechanism has good torque-isotropic degree in the middle area of the working space, so as to determine the task space of the force feedback operating rod.
\end{abstract}

\section{Introduction}

As an interface of human-computer interaction (computer or robot) based on force, force feedback device can collect the movement information of human hands to control a computer or robot as well as output a regular force feedback for the operator, through which the physical state of a computer or robot can be felt by the operator. At present, teleoperation operating technology based on force feedback equipment is widely used by robot systems in outer space, dangerous, radioactive, high temperature, poisonous, undersea environment and other special environment. As it is extremely difficult for professionals to control the robot to complete a task in the above environment, any error in operation can lead to huge losses. Therefore, the teleoperation operating system requires very high accuracy, efficiency and safety performance. As shown in Figure 1, the force interaction technique based on force interaction devices can effectively improve the "telepresence" and "transparency" of teleoperation systems, which can increase force sense information on the basis of visual and auditory channels to help the operator to perceive the unknown environment from the client end, feel like being personally on scene and greatly improve the ability to complete the teleoperation task.

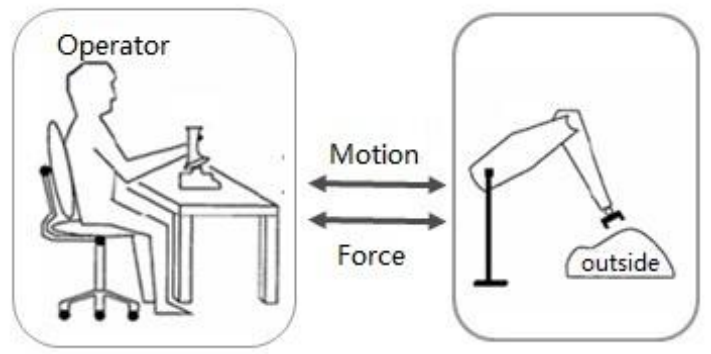

Fig. 1 Schematic diagram of man-machine operation of force feedback operating rod

As the mechanism configuration of the force feedback operating rod determines its working space, dexterity, force transmission performance and so on, the first step in the design of the force feedback operating rod is to select and design the configuration of the mechanism reasonably. There are many kinds of mechanism of force feedback devices, which can be divided into three categories according to the configuration characteristics: series type, parallel type and mixed type. Characteristics of good configuration include high stiffness, high precision, small inertia, large output force / torque, compact structure in accordance with human engineering and so on. By comparing the series-parallel 
mechanism with three rotational degrees of freedom, in this paper, we selected the final configuration scheme of force feedback operating rod with three degrees of freedom and and carried out the modeling and calculation, scale integration and performance analysis.

\section{Configuration Design of the Operating Rod}

\subsection{Comparison and Determination of the Configuration}

Often used as a control input device, the operating rod or joystick is one of the most common human-computer interaction interfaces. According to the operation task, the control signal will be transmitted into the control system through the rod, so that the operator can control the operation of the actuator. The traditional operating rod can be divided into the game operating rod and the industrial operating rod according to the application. These operating rods generally use a configuration of series mechanism as shown in Figure 4.

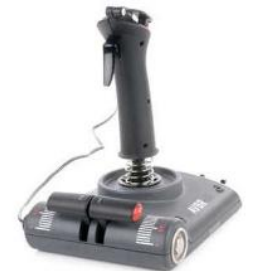

Figure 2 Game operating rod

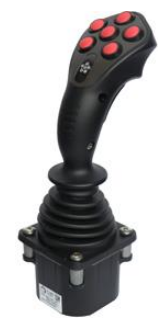

Figure 3 Industrial operating rod

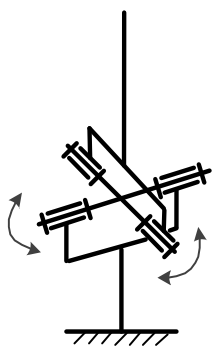

Fig. 4 The configuration of the series mechanism of the operating rod

Although the configuration of series mechanism is simple and the angular displacement collected by which can be directly used as the control signal, it has a lot of shortcomings as a force feedback interaction device. Force feedback devices need to be able to provide driving components for torque output and to be mounted on the revolute pairs of each driving, which is generally a servo motor. If the serial mechanism is used, the motor will move along with the rotating shaft, as the motor quality is rather large, the quality and inertia of the operating end will be increased, which is not conducive to the transparency and reverse driving performance of the force feedback device. At the same time, the moving chain of the series mechanism is generally longer. With larger size and poor stiffness, it is not conducive to the compactness of the device. When designing a force feedback operating rod, mechanism configuration should be designed to meet the three degree of freedom, as all of the driving motor should be installed on the base with a larger working space and better ergonomic characteristics.

To control the gesture of mechanical arm in a special environment, force feedback operating rod proposed in this paper should have three rotational degrees of freedom and can make $\mathrm{X}, \mathrm{Y}, \mathrm{Z}$ coordinate axes in space fixed coordinate system (the coordinate system of force feedback device) couple rotate or sequentially rotate as well as output the regular feedback torque to the operator. The rotation range should be along the $\mathrm{X}, \mathrm{Y}, \mathrm{Z}$ three coordinate axes with relative fixed initial position, rotation range: -45 degrees to +45 degrees; Feedback torque output should be along the $X, Y, Z$ three coordinate axes, the continuous output feedback torque $300 \mathrm{Nmm}$; the envelope size should be less than $200 \mathrm{~mm} * 200 \mathrm{~mm} * 300 \mathrm{~mm}$; attitude angle of operating rod at the real time output end, that is : operating rod angle around the $\mathrm{Y}, \mathrm{X}, \mathrm{Z}$ three coordinate axes, accuracy of 0.1 degree. According to the above requirements, the spherical parallel mechanism (SPM) was selected as the mechanism scheme. Spherical parallel mechanism with three degrees of freedom is a kind of parallel mechanism 
with few degrees of freedom, which is usually used as the robot's shoulder, joint, and so on. With a very good dexterity and stiffness, it can meet the mechanism requirements of the force feedback operating rod.

For the spherical parallel mechanism with three degrees of freedom, the domestic and foreign scholars have carried out a more systematic study as Asada[1] presented a kind of spherical mechanism drivingn by a common axis, Karouia[2] et al. proposed a 3-UPU mechanism, Raffaele[3][4] studied 3-RRS joint joint and 3-URC joint joint as shown in Figure 5, figure 6, Gosselin[5] et al. developed a Agile Eye camera positioning device. In addition, Li Qinchuan et al. [6] proposed a 3-PRS spherical three degree of freedom parallel mechanism, as shown in figure 7; Huang Zhen, etc.[7] Yanshan University, analyzed the kinematic characteristics of a variety of three degrees of freedom by using the anti spiral theory, Including 3-RPS, 3-UPU (RRPRR), 3-PSP, 3-CS and other mechanisms, as shown in Figure 8 to Figure 12 respectively.

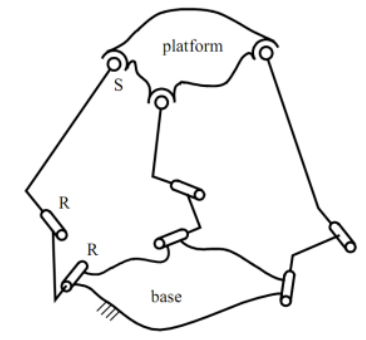

Figure 5 3-RRS joint

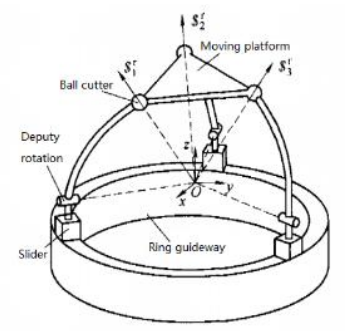

Figure 7 3-PRS spherical parallel mechanism

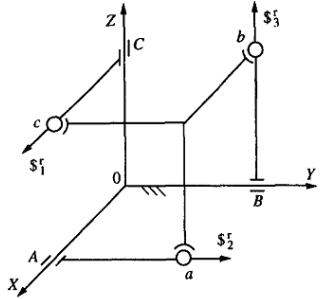

Figure 9 3-CS

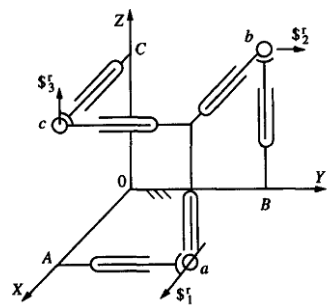

Figure 11 3-PSP

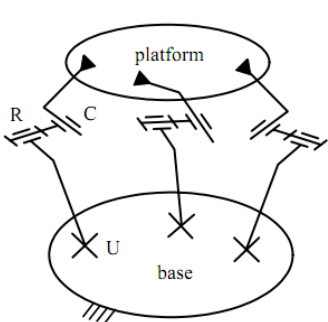

Figure 6 3-URC joint

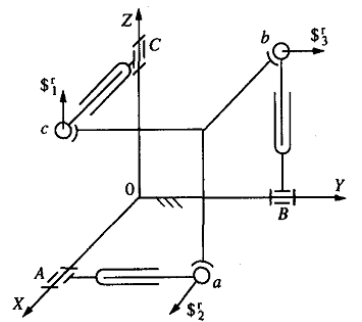

Figure 8 3-RPS

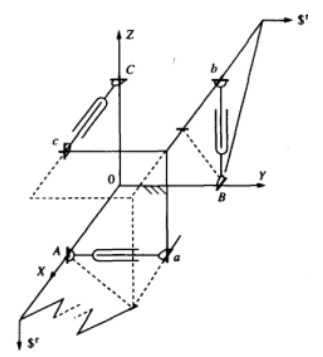

Figure 10 3-UPU

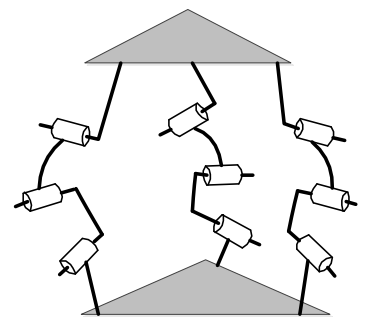

Figure 12 3-RRR spherical parallel mechanism

In summary, as there are many kinds of parallel mechanisms that can achieve the three degree of freedom spherical movement, Factors that should be considered in the design of a mechanism should include compact structure, easy implementation and high reliability and so on. Compared to mobile pair and ball pair, the revolute pair is easier to implement, and the frictional resistance is smaller, 
therefore, 3-RRR mechanism, a simple and reliable mechanism is selected as the configuration of force feedback operating rod in the design of this paper, as shown in Figure 12. 3-RRR spherical parallel mechanism has the following advantages compared with the other three degree of freedom mechanisms:

Under the same working space and output impedance, three degree of freedom spherical parallel mechanism with small size, simple and compact structure;

2) The structure stiffness is high making it not easy to bend and deform and with high external load bearing capacity;

3) None Error accumulation and amplification effect, high precision;

4) As a fully symmetrical parallel mechanism, the spherical mechanism has better isotropic properties;

5) As moving chain is short, the driving device is fixed on the base, so the operation end is light, the motion inertia is small, and the dynamic response performance of the high velocity operation is good;

6) Rotational degrees of freedom with three rotating shafts into one point, similar with human body's joint, shoulder, and so on, in accordance with the principles of human engineering.

\subsection{Configuration Scheme of the Operating Rod}

The force feedback operating rod configuration is shown in Figure 13. Originally proposed by Asada and Cro Granito [8], this kind of 3-RRR spherical parallel structure then got the attention and research of many scientists. (Craver 1989[9], Gosselin and Angeles 1989[10]), and has been widely used. In 1994, using 3-RRR spherical parallel mechanism, Gosselin and Hamel [11] developed the famous device, Agile Eye. The miniature camera installed of the end can rotate flexibly along the three rotating shafts, the working space of which is larger than the human eye. Through the size optimization and analysis kinetic, the mechanism obtained a very high accuracy. Using human body engineering characteristics of 3-RRR parallel mechanism, Malosio developed a robot system to meet the requirements of ankle rehabilitation.

The mechanism has three rotational degrees of freedom, the rotating shafts of which meet in the center of rotation of the end actuator. This configuration is similar to the human body's joint, shoulder, etc. and in accordance with the principles of human engineering. The operating rod is designed on the platform. The feedback force for the operator is realized by the permanent magnet synchronous motor driving joint with less torque ripple. High-precision absolute encoder is mounted on three degree of freedom joints, helping operator to find the reference point of kinematics solution and kinematic solution accuracy of the mechanism.

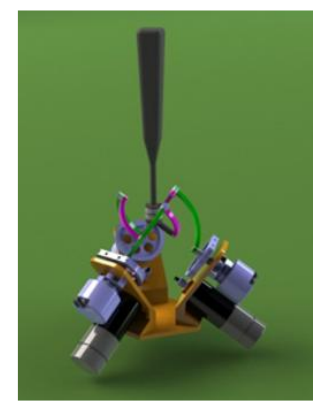

Fig. 13 CAD model of the 3-RRR spherical parallel force feedback operating rod

\section{Analysis of Kinematics and Statics of 3-RRR Spherical Parallel Mechanism}

3-RRR mechanism consists of three branch chains, a moving platform, and a static platform. Each branch chain is composed of two connecting rods and three revolute pairs. The static platform of the mechanism is the frame and the moving platform is connected with the operating rod. In order to obtain the control signal to input the operator, we have to obtain the rotation angle of the moving platform around the three axis, that is, attitude angle $(\phi, \theta, \psi)$, represented by Z-X-Y Euler angle in this paper. Firstly, we model the 3-RRR spherical parallel mechanism. 


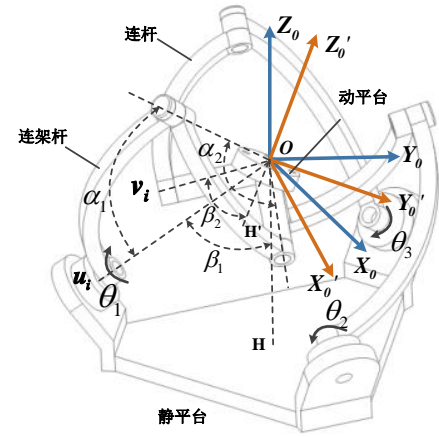

Fig. 14 3-RRR mechanism and its structure parameters

The three branch chains of the 3-RRR mechanism have the same structure as shown in Figure 14. The static platform coordinate system is defined as $O X_{0} Y_{0} Z_{0}$ while moving platform coordinate system is $O X_{0}{ }^{\prime} Y_{0}{ }^{\prime} Z_{0}{ }^{\prime}$. The coordinate origin is located in the center of rotation, which is defined as $O$. $Z_{0}$ axis direction is for the normal direction of the static platform and $Z_{0}{ }^{\prime}$ axis is for the moving platform. At the same time, the axis directions of three revolute pairs of each branch chain $\boldsymbol{u}_{\boldsymbol{i}}, \boldsymbol{w}_{\boldsymbol{i}}, \boldsymbol{v}_{\boldsymbol{i}}$ are defined as directions of driving shaft, hinge of frame liking rod and connecting rod, and hinge of connecting rod and moving platform. Each branch chain has four structure parameters:

$\alpha 1$-angle between the two ends of the connecting rod;

$\alpha 2$-angle between axis of the two ends of connecting rod;

$\beta 1$-angle between joint angle axis ui and static platform normal;

$\beta 2$--angle between axis vi and moving platform normal $\mathrm{OH}^{\prime}$.

\subsection{Inverse Kinematic Analysis}

The kinematic analysis of mechanism is the foundation of motion control, and the premise of static solution. The basic method of inverse kinematics analysis is to obtain the rotation matrix of the dynamic coordinate system to the fixed coordinate system through the D-H coordinate system, and then write the constraint Equations according to the geometric relations, and finally solve the Equation.

First of all, the D-H method is used to establish the coordinate system of the mechanism. Then establish a $O X_{0} Y_{0} Z_{0}$ coordinate system around the center of rotation $O$, as shown in Figure $41 . Z_{0}$ axis and vertical line $\mathrm{OH}$ is coincident with each other. The three rotation axes of each branch chain are respectively defined as $\boldsymbol{u}_{i}, \boldsymbol{w}_{\boldsymbol{i}}, \boldsymbol{v}_{\boldsymbol{i}}(i=1,2,3)$. Establish the coordinate system $O X_{i 1} Y_{i 1} Z_{i 1}$, $O X_{i 2} Y_{i 2} Z_{i 2}, O X_{i 3} Y_{i 3} Z_{i 3}$ of each connecting rod according to the D-H method. $Z_{i 1}$ is coincident with $\boldsymbol{u}_{i}$, $X_{i l}$ perpendicular to $Z_{i 1}$ and $O H, Y_{i l}$ in accordance with the right-hand rule; $Z_{i 2}$ and $Z_{i 3}$ are defined to be coincident with $\boldsymbol{w}_{i}$ and $\boldsymbol{v}_{i}$.

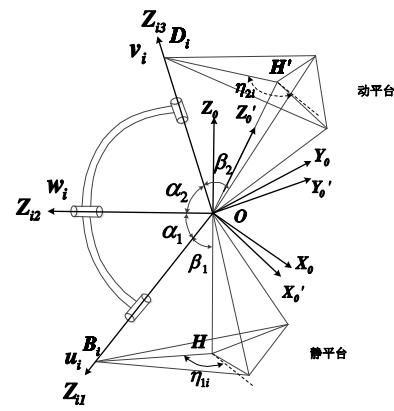

Figure 15 The establishment of coordinate system

The Rotation matrix from coordinate system $O X_{0} Y_{0} Z_{0}$ to coordinate system $O X_{i 1} Y_{i 1} Z_{i 1}$ is defined as $\boldsymbol{R}_{01 i}$. 


$$
\boldsymbol{R}_{01 i}=\left[\begin{array}{lll}
-s \eta_{1 i}, & c \eta_{1 i} c \beta_{1}, & c \eta_{1 i} s \beta_{1} \\
c \eta_{1 i}, & s \eta_{1 i} c \beta_{1}, & s \eta_{1 i} s \beta_{1} \\
0, & s \beta_{1}, & -c \beta_{1}
\end{array}\right], \quad i=1,2,3
$$

Thereof, $\eta_{1 i}, \eta_{2 i}$ is the torsion angle of the structure of connecting rod of the static platform and the moving platform, as shown in Figure 15. $\eta_{1 i}$ is the included angle between the projection of $H B_{i}$ and $X_{0}$ on the static platform; $\eta_{2 i}$ is the included angle between the projection of $H D_{i}$ and $X o^{\prime}$ on the moving platform; For the 3-RRR mechanism designed in this paper, $\eta_{1 i}=2 \pi(i-1) / 3$, $\eta_{2 i}=2 \pi(i-2) / 3, i=1,2,3$;

The Rotation matrix from coordinate system $O X_{i 1} Y_{i 1} Z_{i 1}$ to coordinate system $O X_{i 2} Y_{i 2} Z_{i 2}$ is defined as $\boldsymbol{R}_{12 i}$.

$$
\boldsymbol{R}_{12 i}=\left[\begin{array}{ccc}
c \theta_{i}, & -s \theta_{i} c \alpha_{1}, & s \theta_{i} s \alpha_{1} \\
s \theta_{i}, & c \theta_{i} c \alpha_{1}, & -c \theta_{i} s \alpha_{1} \\
0, & s \alpha_{1}, & c \alpha_{1}
\end{array}\right], \quad i=1,2,3 ;
$$

Define the fixed coordinate system of the moving platform as $O X_{0}{ }^{\prime} Y_{0}{ }^{\prime} Z_{0}{ }^{\prime}$ and Z-X-Y Euler angle as $(\phi, \theta, \psi)$, then Euler matrix is

$$
\boldsymbol{R}_{E}=\left[\begin{array}{ccc}
c \phi c \psi-s \phi s \theta s \psi & -s \phi c \theta & c \phi s \psi+s \phi s \theta c \psi \\
s \phi c \psi+c \phi s \theta s \psi & c \phi c \theta & s \phi s \psi-c \phi s \theta c \psi \\
-c \theta s \psi & s \theta & c \theta c \psi
\end{array}\right]
$$

The Rotation matrix from coordinate system $O X_{0}{ }^{\prime} Y_{0}{ }^{\prime} Z_{0}{ }^{\prime}$ to coordinate system $O X_{i 3} Y_{i 3} Z_{i 3}$ is defined as $\boldsymbol{R}_{0^{\prime} 3 i}$.

$$
\boldsymbol{R}_{0^{\prime 3 i}}=\left[\begin{array}{lll}
-s \eta_{2 i}, & c \eta_{2 i} c \beta_{2}, & c \eta_{2 i} s \beta_{2} \\
c \eta_{2 i}, & s \eta_{2 i} c \beta_{2}, & s \eta_{2 i} s \beta_{2} \\
0, & s \beta_{2}, & -c \beta_{2}
\end{array}\right], \quad i=1,2,3
$$

Vector $\boldsymbol{w}_{i}, \boldsymbol{v}_{i}$ in the fixed coordinate system $O X_{0} Y_{0} Z_{0}$ can be expressed as follows:

$$
\begin{aligned}
& \boldsymbol{w}_{i}=\boldsymbol{R}_{01 i} \boldsymbol{R}_{12 i} \boldsymbol{e} \\
& \boldsymbol{v}_{i}=\boldsymbol{R}_{E} \cdot \boldsymbol{R}_{0 \prime 3 i} \boldsymbol{e}
\end{aligned}
$$

Thereof, $\boldsymbol{e}=[0,0,1]^{T}$

Constraint Equations can be obtained by geometric relations:

$$
\boldsymbol{w}_{i}^{T} \boldsymbol{v}_{i}=\cos \alpha_{2}, \quad i=1,2,3
$$

The process of kinematics inverse solution of 3-RRR mechanism is: Given the position and attitude of the moving platform $(\phi, \theta, \psi)$, find the angle $\left(\theta_{1}, \theta_{2}, \theta_{3}\right)$ of the driving joint, then solve the Equation (7) and we can get the inverse kinematics solution of the mechanism.

\subsection{Velocity Jacobi Matrix}

Jacobi matrix is used to describe the velocity relation of joint space and operation space of a mechanism, therefore, the Jacobi matrix is also known as the generalized transmission ratio. Based on the reference [12], in the following, we will find the Jacobi matrix of the 3-RRR spherical parallel mechanism.

Given that the angular velocity of the end of the mechanism is $\omega$, driving joint angular velocity is $\dot{\boldsymbol{s}}$, therefore, the velocity relation of 3 -RRR spherical parallel mechanism can be described as:

$A \omega=B \dot{s}$

Therefore, $\boldsymbol{A}$ is the Type 1 Jacobi matrix, $\boldsymbol{B}$ is the Type 2 Jacobi matrix, 


$$
\begin{aligned}
& \boldsymbol{A}=\left[\begin{array}{l}
\left(\boldsymbol{w}_{1} \times \boldsymbol{v}_{1}\right)^{T} \\
\left(\boldsymbol{w}_{2} \times \boldsymbol{v}_{2}\right)^{T} \\
\left(\boldsymbol{w}_{3} \times \boldsymbol{v}_{3}\right)^{T}
\end{array}\right] \\
& \boldsymbol{B}=\operatorname{diag}\left(\boldsymbol{u}_{i} \times \boldsymbol{w}_{i}\left[\boldsymbol{v}_{i}\right) \quad i=1,2,3 ;\right.
\end{aligned}
$$

$\boldsymbol{J}$ is velocity mapping between input and output of the mechanism $(\dot{\boldsymbol{s}}=\boldsymbol{J} \boldsymbol{\omega})$, the velocity Jacobean matrix, and

$$
\boldsymbol{J}=\boldsymbol{B}^{-1} \boldsymbol{A}
$$

In the control, we need to use the Euler angle for differential of time, so we introduce the conversion matrix $\boldsymbol{R}$,

$$
\omega=\boldsymbol{R} \dot{\boldsymbol{t}}
$$

Therefore, $\boldsymbol{t}$ is Euler angle vector, $\boldsymbol{t}=[\phi, \theta, \psi]^{T}$;

$$
\boldsymbol{R}=\left[\begin{array}{ccc}
0 & \cos \phi & -\cos \theta \sin \phi \\
0 & \sin \phi & \cos \theta \cos \phi \\
1 & 0 & \sin \theta
\end{array}\right]
$$

Thus,

$$
\dot{\boldsymbol{s}}=\boldsymbol{J} \dot{\boldsymbol{t}}=\boldsymbol{J}_{\boldsymbol{e}} \dot{\boldsymbol{t}}
$$

In the formula, $\boldsymbol{J}_{\boldsymbol{e}}=\boldsymbol{J} \boldsymbol{R}$ is the extended Jacobian matrix.

\subsection{Positive Kinematic Analysis}

Given that the driving joint angle is $\left(\theta_{1}, \theta_{2}, \theta_{3}\right)$, it is very complex for us to use Equation (7) to find the position and attitude $(\phi, \theta, \psi)$ of the moving platform, not only difficult to solve, but generally we can not find the only solution. In order to simplify the solution, for the process of positive solution, we define an additional moving platform coordinate system $O X_{2} Y_{2} Z_{2}$, axis $Z_{2}$ along the direction of vector $\boldsymbol{v}_{1}$ while axis $X_{2}$ perpendicular to the plane between $Z_{2}$ and axis defined by $\boldsymbol{v}_{1}$, $\boldsymbol{v}_{2}$, and axis $Y_{2}$ in accordance with the right-hand rule. At this time, vector $\boldsymbol{v}_{1}, \boldsymbol{v}_{2}, \boldsymbol{v}_{3}$ in coordinate system $\mathrm{OX}_{2} Y_{2} Z_{2}$ expressed as:

$$
\begin{aligned}
& v_{1}^{\prime}=[0,0,1]^{T}, \\
& v_{2}^{\prime}=\left[\sin \gamma_{2}, 0, \cos \gamma_{2}\right]^{T}, \\
& v_{3}^{\prime}=\left[\cos \psi_{2} \sin \left(\gamma_{2} / 2\right), \sin \psi_{2}, \cos \psi_{2} \cos \left(\gamma_{2} / 2\right)\right]^{T}
\end{aligned}
$$

Therefor, $\gamma_{2}=2 \arcsin \left(\frac{\sqrt{3} \sin \beta_{2}}{2}\right)$

$$
\psi_{2}=\beta_{2}+\arctan \left(\frac{\sin \left(\gamma_{2} / 2\right)}{\sqrt{3-4 \sin ^{2}\left(\gamma_{2} / 2\right)}}\right)
$$

We define Z-Y-Z Euler angle of additional moving coordinate system $\mathrm{OX}_{2} \mathrm{Y}_{2} \mathrm{Z}_{2}$ and fixed coordinate system $O X_{0} Y_{0} Z$ as $o \phi_{1}, \phi_{2}, \phi_{3}$

Rotation matrix can be expressed as:

$$
Q=Q_{1} Q_{2} Q_{3}
$$

$$
\text { Therefor, } \begin{aligned}
\boldsymbol{Q}_{1} & =\left[\begin{array}{ccc}
\cos \phi_{1} & -\sin \phi_{1} & 0 \\
\sin \phi_{1} & \cos \phi_{1} & 0 \\
0 & 0 & 1
\end{array}\right] \\
\boldsymbol{Q}_{2} & =\left[\begin{array}{ccc}
\cos \phi_{2} & 0 & \sin \phi_{2} \\
0 & 1 & 0 \\
-\sin \phi_{2} & 0 & \cos \phi_{2}
\end{array}\right]
\end{aligned}
$$


$\boldsymbol{Q}_{3}=\left[\begin{array}{ccc}\cos \phi_{3} & -\sin \phi_{3} & 0 \\ \sin \phi_{3} & \cos \phi_{3} & 0 \\ 0 & 0 & 1\end{array}\right]$

$v_{i}$ in the fixed coordinate system $O X_{0} Y_{0} Z_{0}$ can be expressed as follows:

$\boldsymbol{v}_{i}=\boldsymbol{R}_{011} \cdot \boldsymbol{R}_{121} \cdot \boldsymbol{Q} \cdot \boldsymbol{v}_{i}^{\prime} \quad i=1,2,3$

Substitute the Expression (16) into Equation (7),

Therefore, the Euler angle $\phi_{2}$ can be obtained, and then substitute $\phi_{2}$ into the equation to be solved, after simplification:

$$
\begin{aligned}
& I_{1} \sin \phi_{1}+I_{2} \cos \phi_{1}+I_{3}=0 \\
& J_{1} \sin \phi_{1}+J_{2} \cos \phi_{1}+J_{3}=0
\end{aligned}
$$

Therefor,

$$
\begin{aligned}
& I_{1}=A_{1} \sin \phi_{3}+B_{1} \cos \phi_{3}+F_{1} \\
& I_{2}=A_{2} \sin \phi_{3}+B_{2} \cos \phi_{3}+F_{2} \\
& I_{3}=A_{3} \sin \phi_{3}+B_{3} \cos \phi_{3}+F_{3} \\
& J_{1}=C_{1} \sin \phi_{3}+D_{1} \cos \phi_{3}+G_{1} \\
& J_{2}=C_{2} \sin \phi_{3}+D_{2} \cos \phi_{3}+G_{2} \\
& J_{3}=C_{3} \sin \phi_{3}+D_{3} \cos \phi_{3}+G_{3}
\end{aligned}
$$

Coefficients $A_{i}, B_{i}, C_{i}, D_{i}, F_{i}, G_{i}$ in these expressions are the functions of structure parameters and driving angle. As the expression is complex, we omitted it here.

Through formula (17)(18), there are

$$
\begin{aligned}
& \sin \phi_{1}=\frac{-I_{3} J_{2}+I_{2} J_{3}}{-I_{2} J_{1}+I_{1} J_{2}} \\
& \cos \phi_{1}=\frac{I_{3} J_{1}-I_{1} J_{3}}{-I_{2} J_{1}+I_{1} J_{2}}
\end{aligned}
$$

Substitute formula (19) (20), there are

$$
\begin{aligned}
& -I_{2}^{2} J_{1}^{2}+I_{3}^{2} J_{1}^{2}+2 I_{1} I_{2} J_{1} J_{2}-I_{1}^{2} J_{2}^{2}+I_{3}^{2} J_{2}^{2}-2 I_{1} I_{3} J_{1} J_{3}-2 I_{2} I_{3} J_{2} J_{3}+I_{1}^{2} J_{3}^{2}+I_{2}^{2} J_{3}^{2}=0 \\
& \left(I_{1} J_{2} \neq I_{2} J_{1}\right)
\end{aligned}
$$

Introduce variable $t$,

Substitute formula (21), there are

$$
\sum_{i=0}^{8} N_{i} t^{i}=0
$$

Through formula (22), we can find, thus by formula (19)(20), we can solve, and through formula (15), we can work out the rotation matrix from the moving platform to the static platform coordinate system so as to solve the solution of the actual position and attitude.

\subsection{Static Analysis}

The purpose of static analysis is to find the mapping relationship between the joint space and the operation space. In order to implement force control of the end of the force feedback operating rod, when the end of the 3-RRR spherical parallel mechanism is known to be stressed, we have to solve the driving torque of each driving joint, so as to control the servo motor to output current of the corresponding size.

Given that the gravity of the mechanism member is 0 , taking into account the relationship between the end torque of the mechanism and the driving force of the joint, define external torque of the end of the operating rod as $\boldsymbol{M}$, and the torque of the driving joint is $\boldsymbol{\tau}$,

According to the principle of virtual work, there are 


$$
\boldsymbol{M}^{T} \dot{\boldsymbol{t}}=\boldsymbol{\tau}^{T} \dot{\mathbf{s}}
$$

Substitute the formula(14) into (23), there are

$$
\boldsymbol{\tau}=\boldsymbol{J}_{e}{ }^{-T} \boldsymbol{M}=\boldsymbol{J}_{m} \boldsymbol{M}
$$

Therefore, $\boldsymbol{J}_{\boldsymbol{m}}=\boldsymbol{J}_{\boldsymbol{e}} \boldsymbol{T}^{-T_{\mathrm{i}}}$ the Jacobi matrix, expressing the torque mapping relation from the end to the joint.

\section{Analysis of Dexterity and Force Transfer Performance of the Operating Rod Mechanism}

\subsection{Analysis of Dexterity of the Mechanism}

Reflecting the overall transmission performance of the mechanism, the dexterity is an important indicator to evaluate the performance of the mechanism [13]. Based on the method of Gosselin [12], the reciprocal of condition number in Jacobi matrix of 3-RRR spherical parallel mechanism is defined as the dexterity index $\xi$.

$$
\xi=1 / \kappa(\boldsymbol{J})
$$

Thereof,

$$
\kappa(\boldsymbol{J})=\|\boldsymbol{J}\| \cdot\left\|\boldsymbol{J}^{-1}\right\|
$$

$\|\mathbf{J}\|$ is the Euclidean norm of Jacobi matrix;

$$
\|\boldsymbol{J}\|=\sqrt{\operatorname{tr}\left(\boldsymbol{J} \boldsymbol{W} \boldsymbol{J}^{T}\right)}
$$

$\mathrm{W}$ is the weight matrix,

$\boldsymbol{W}=\operatorname{diag}(1 / 3,1 / 3,1 / 3)$

Defining the global dexterity index of the mechanism in working space [14]:

$$
\eta=\frac{\int_{w} \xi d w}{\int_{w} d w}
$$

$w$ is the working space.

The global dexterity index of the mechanism is related to its size parameters $\left(\left[\alpha_{1}, \alpha_{2}, \beta_{1}, \beta_{2}\right]\right)$. It proved that [15], with the same dexterity isotropic, if the vector $\boldsymbol{u}, \boldsymbol{v}, \boldsymbol{w}$ orthogonal to each other, the input and output velocity mapping of the mechanism achieves the maximum value making the transmission performance of the mechanism the best, that is, the size parameter of the mechanism is orthogonal:

$$
\alpha_{1}=90^{\circ}, \alpha_{2}=90^{\circ} \text {; }
$$

Based on the above orthogonal condition, in order to obtain the optimal configuration, we can search the parameter as shown in Figure 16 when the global dexterity index $\eta$ achieves maximum value. On the basis of analysis, when $\beta_{1}=\beta_{2}=54^{\circ}$, the global dexterity index achieves maximum value, $\eta_{\max }=0.8906$, so the size parameter of 3-RRR spherical parallel mechanism is:

$\alpha_{1}=90^{\circ}, \alpha_{2}=90, \beta_{1}=54^{\circ}, \beta_{2}=54^{\circ}$;

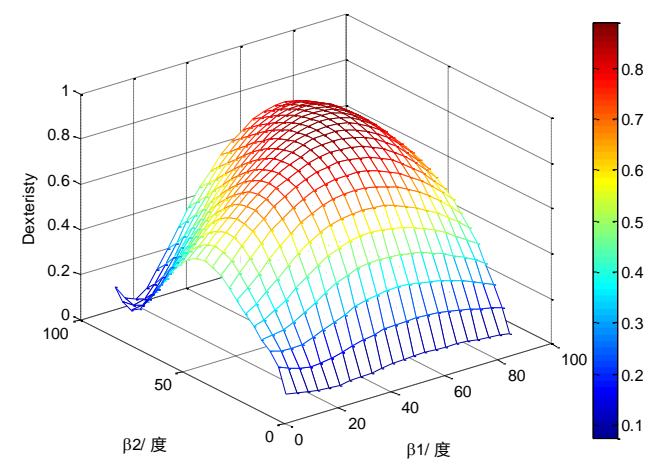

Fig. 16 Effect of $\beta 1, \beta 2$ on global dexterity 


\subsection{Analysis of Force Transfer Performance of the Mechanism}

The isotropic problem of the output force / torque of an equipment the degree of isotropy of the output force in any direction and output torque around an arbitrary axis of the end of the operation when the device in a position. Force / torque isotropy performance is an important performance index of parallel mechanism and has a great significance of force transmission accuracy measurement and mechanism design optimization

For the force feedback operating rod designed in this paper, control performance of the feedback force and the force transfer performance of the mechanism is closely related to each other. Studies on the torque transmission isotropic properties is helpful to determine an area with better transmission performance in working space, thereby to optimize the task space.

Formula (24) can be converted to

$$
\boldsymbol{M}=\boldsymbol{J}_{m}^{-1} \boldsymbol{\tau}
$$

Formula (2.28) shows that the torque isotropy performance depends on the Jacobi matrix. Given that the joint driving torque mode is 1, i.e.:

$$
\|\boldsymbol{\tau}\|=\sqrt{\boldsymbol{\tau}^{T} \boldsymbol{\tau}}=1
$$

When the mechanism is not in a singular position, there is a quadratic equation as follows:

$$
K_{M}=\left|\sigma_{3 M} / \sigma_{1 M}\right|
$$

Formula (30) shows that the output torque $\boldsymbol{M}$ is located on an ellipsoid when the mode of the joint driving torque vector is 1 . Given that $\sigma_{1}$ and $\sigma_{3} M$ are the maximum and minimum singular value of the matrix, and define the isotropy as:

$$
K_{M}=\left|\sigma_{3 M} / \sigma_{1 M}\right|
$$

Formula (31) is equivalent to the reciprocal condition number of force Jacobi matrix $\boldsymbol{J}_{m}$ :

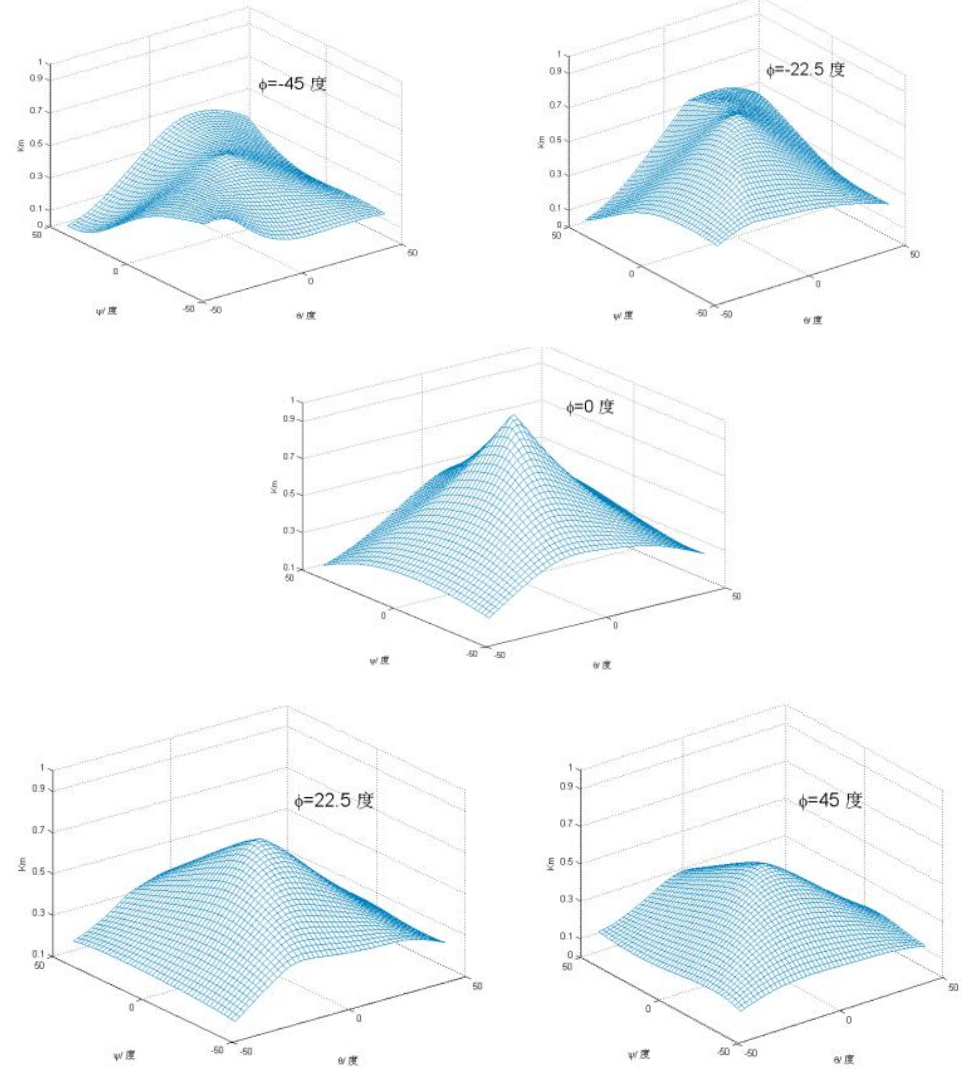

Figure 17 The attitude angle from -45 degrees to 45 degrees, the distribution of torque isotropy in $\theta$ $\psi$ plane 
The range of value of $K_{M}$ is $0 \leq K_{M} \leq 1$. When $K_{M=1}$, the ellipsoid changes into a sphere, at this time, the isotropy of output torque of the end is the same. For the designed 3-RRR spherical parallel mechanism, we take one attitude angle as the control variable in the end attitude space. In the plane of the other two attitude angles, we carry out a uniform scan, and calculate the isotropy of torque at each point. The calculated torque isotropic properties are shown in Figure 17, and there are

The isotropy of output torque of the 3-RRR spherical mechanism is related to the attitude of the end. In middle region of the attitude space, the torque isotropy has a larger value. When all the three attitude angles are $0, K_{M}$ is about 0.9995 , close to 1 , showing that the torque isotropy is better when the operating rod is positioned at the middle position.

The closer to the edge of the attitude space, the worse the isotropy performance of the torque, so when determining the task space of the mechanism, we should be in the center of the working space as far as possible. For 3-RRR spherical parallel mechanism, the theoretical work space is a spherical surface, and the center of the sphere is the intersection of each axes. On the basis of design requirements of force feedback operating rod, starting from the zero, the motion range of $\pm 45^{\circ}$ of each attitude angles is selected as a task space, and the task space based on Matlab simulation as shown in Figure 18.

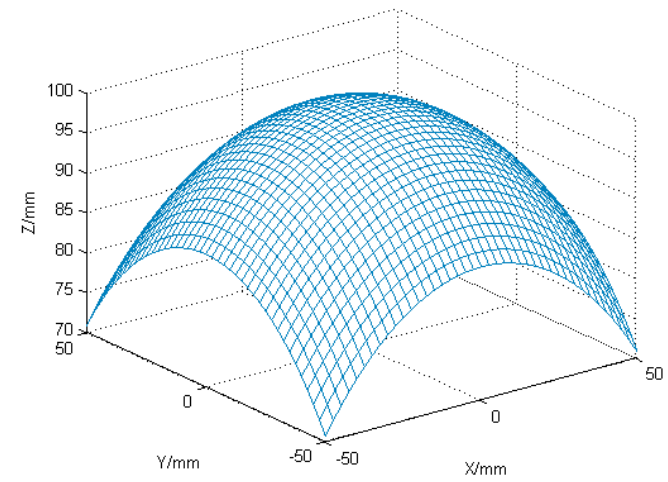

Figure 18 Task space of 3-RRR spherical parallel mechanism

\section{Conclusion}

Based on the design requirements of the force feedback operating rod, in this paper, we designed a kind of force feedback operating rod based on 3-RRR spherical parallel mechanism, integrated the size of the mechanism and analyzed its force transfer performance. On this basis, we determined task space of the force feedback operation by MATLAB simulation analysis. In the middle area of the working space, with good torque isotropic degrees and ergonomics characteristics, the mechanism can be used in a variety of teleoperation manipulator control, providing a good engineering use value.

\section{Reference}

[1] Asada H, Granito J A C. Kinematic and static characterization of wrist joints and their optimal design[C]//Robotics and Automation. Proceedings. 1985 IEEE International Conference on. IEEE, 1985, 2: 244-250.

[2] Karouia M, Hervé J M. A three-dof tripod for generating spherical rotation [M]//Advances in Robot Kinematics. Springer Netherlands, 2000: 395-402.

[3] Di Gregorio R. The 3-RRS Wrist: A New, Very Simple and Not Over-Constrained Spherical Parallel Manipulator[C]//ASME 2002 International Design Engineering Technical Conferences and Computers and Information in Engineering Conference. American Society of Mechanical Engineers, 2002: 1193-1199.

[4] Di Gregorio R. Kinematics of a new spherical parallel manipulator with three equal legs: The 3 URC wrist [J]. Journal of Robotic Systems, 2001, 18(5): 213-219. 
[5] Gosselin C M, Hamel J F. The agile eye: a high-performance three-degree-of-freedom cameraorienting device[C]//Robotics and Automation, 1994. Proceedings, 1994 IEEE International Conference on. IEEE, 1994: 781-786.

[6] Li Qinchuan, Wu Chuanyu, Shen Weiping, and so on. New 3-PCRNS spherical 3 degree of freedom parallel mechanism [J]. Chinese Journal of Mechanical Engineering, 2007, 42 (11): 4448.

[7] Huang Z, Fang Y F. Kinematic characteristics analysis of 3 DOF in-parallel actuated pyramid mechanism [J]. Mechanism and Machine Theory, 1996, 31(8): 1009-1018.

[8] Asada H, and Cro Granito J. A. Kinematic and static characterization of wrist joints and their optimal design. In Proc. of the IEEE International Conference on Robotics and Automation, 1985. 244-250.

[9] Craver W.M. Structural analysis and design of a three-degree-of-freedom robotic shoulder module [D]. Master's Thesis, The University of Texas at Austin. 1989.

[10] Gosselin C.M. and Angeles J. The optimum kinematic design of a spherical three- degree- offreedom parallel manipulator. ASME J. Mech. Trans Automat. Design. 1989, 111(2):202-207.

[11]Gosselin C.M. and Hamel J.-F. The agile eye: a high-performance three-degree-of-freedom cameta-orienting device. Proc. of the IEEE Inter. Conf. on Robotics and Automation, Los Alamitos, 1994: 781-786.

[12]Birglen L, Gosselin C, Pouliot N, et al. SHaDe, a new 3-dof haptic device[J]. Robotics and Automation, IEEE Transactions on, 2002, 18(2): 166-175.

[13] San Hingjun, Zhong Shisheng, Wang Zhixing. dexterity analysis of a new type of parallel mechanism with 4 degrees of freedom

[14] Gosselin C, Angeles J. A global performance index for the kinematic optimization of robotic manipulators [J]. Journal of Mechanical Design, 1991, 113(3): 220-226.

[15]Huang Tian, Zeng Xianjing. The whole parameter analysis and scale integration of the spherical parallel mechanism with equal tip cone angle 3 degrees of freedom spherical parallel mechanism [J]. Chinese Journal of Mechanical Engineering, 2000, 36 (8): 15-19.

The Author: Zhiming Wei, From Qinghai Province, mainly research on Space Electronics and Mechatronics, email:castwzm@163.com. 